\title{
The quantum inequalities do not forbid spacetime shortcuts
}

\author{
S. Krasnikov \\ The Central Astronomical Observatory at Pulkovo
}

(Dated:)

\begin{abstract}
A class of spacetimes (comprising the Alcubierre bubble, Krasnikov tube, and a certain type of wormholes) is considered that admits 'superluminal travel' in a strictly defined sense. Such spacetimes (they are called 'shortcuts' in this paper) were suspected to be impossible because calculations based on 'quantum inequalities' suggest that their existence would involve Planck-scale energy densities and hence unphysically large values of the 'total amount of negative energy' $E_{\text {tot }}^{-}$.

I argue that the spacetimes of this type may not be unphysical at all. By explicit examples I prove that: 1) the relevant quantum inequality does not (always) imply large energy densities; 2) large densities may not lead to large values of $E_{\text {tot }}^{-}$; 3) large $E_{\text {tot }}^{-}$being physically meaningless in some relevant situations, does not necessarily exclude shortcuts.
\end{abstract}

\section{INTRODUCTION}

Suppose the distance from the Earth to a star, found by usual astronomical methods (by measuring the parallax, say), is 100 light years. Suppose also that (in agreement with all that we know) no body can move faster than light. It is then tempting to conclude that a spaceship sent to that star cannot return sooner than in $200 \mathrm{yr}$. However, such a conclusion may be too hasty. The point is that the quantity $D_{p}$, which defines the travel time, and the quantity $D_{a}$ measured by the parallax, in general relativity (in contrast with special relativity) are not the same, even though, duly defined, they both deserve the name 'distance'. In practice, some approximately flat (and pretty narrow) region $\mathcal{R}$ is considered, comprising the Earth, the star, and a geodesic connecting them. $D_{a}$ then is defined by that geodesic as if $\mathcal{R}$ were a part of the Minkowski space. That the thus defined $D_{a}$ may be much greater than $D_{p}$ can be seen already from the fact that beyond $\mathcal{R}$ a short wormhole may occur, which connects the vicinities of the Earth and of the star (as we shall see in a moment a non-trivial topology is not essential for the matter in discussion). A spaceship then can 
take a short cut through the wormhole and thus make the trip faster than light. Of course the words 'faster than light', used in such a context do not mean that the spaceship locally (when the notion of speed is well defined) moves faster than a passing photon. Actually in the above example we compared two different spacetimes - the real world with a wormhole and a fictitious Minkowski space by which we erroneously described our world - and found that the travel time of a spaceship in the former is less than that of a photon in the latter.

Generalizing the above example (in the next section we briefly discuss two other possible ways to give a precise meaning to the words 'faster-than-light' in application to non-tachyonic objects) we introduce the following notion.

Definition. Let $C$ be a timelike cylinder $\sum_{i=1}^{3} x_{i}^{2} \leqslant c^{2}$ in the Minkowski space $\mathbb{L}^{4}$. A globally hyperbolic spacetime $M$ is a shortcut if there are a region $U \subset M$, an isometry $\varkappa:\left(\mathbb{L}^{4}-C\right) \rightarrow U$, and a pair of points $p, q \in \mathbb{L}^{4}-C$ such that

$$
p \nprec q, \quad \varkappa(p) \preccurlyeq \varkappa(q) .
$$

In other words we call $M$ a shortcut if it can be obtained from the Minkowski space $\mathbb{L}^{4}$ by replacing a flat cylinder $C \subset \mathbb{L}^{4}$ with something else so that some spacelike separated (in $\left.\mathbb{L}^{4}\right)$ points are causally connected in $M$.

Examples. Consider a plane $\mathbb{R}^{2}$ with the metric

$$
\mathrm{d} s^{2}=\mathrm{d} r^{2}+R^{2}(r) \mathrm{d} \phi^{2},
$$

where

$$
r \geqslant D_{a}-d, \quad \phi=\phi+2 \pi,\left.\quad R\right|_{r<D_{a}}=r-D_{a}+d,\left.\quad R\right|_{r>D_{a}+\delta}=r .
$$

The plane is flat except in a thin annulus $D_{a}<r<D_{a}+\delta$. However, an observer at $r=D_{a}+\delta, \phi=0$ is much closer $-2(d+\delta)$ against $2\left(D_{a}+\delta\right)$ - to the diametrically opposite point $r=D_{a}+\delta, \phi=\pi$ than if the whole plane were flat. A four-dimensional generalization of such a spacetime

$$
\mathrm{d} s^{2}=\mathrm{d} t^{2}-\mathrm{d} r^{2}-R^{2}(r)\left(\mathrm{d} \theta^{2}+\sin ^{2} \theta \mathrm{d} \phi^{2}\right)
$$

is shown in figure 1a. The metric inside the cylinder $Z$ is as flat as the metric outside, but the null cones are 'more open' (this of course is a coordinate effect and would not take place, say, in the spherical coordinates (1)) and thus curves like $\gamma_{A}$ are timelike, though they would be spacelike, if the metric were Minkowski in the whole spacetime. To use the described 


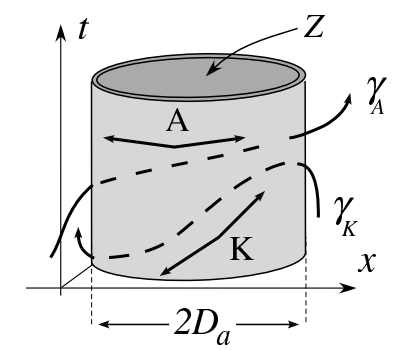

(a)

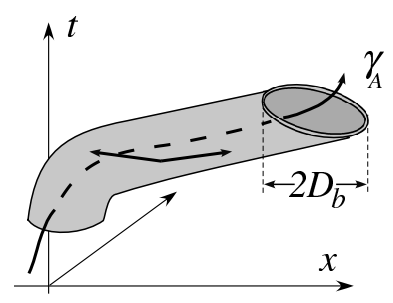

(b)

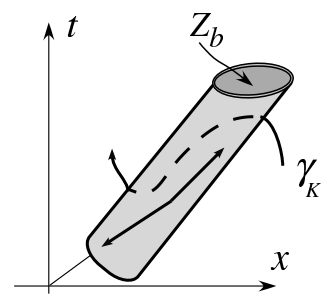

(c)

FIG. 1: The domains bounded by the gray walls may be flat and, nevertheless, differ significantly from $C$. The pairs $\mathrm{A}$ and $\mathrm{K}$ of null vectors correspond to Alcubierre and Krasnikov metrics, respectively.

phenomenon for interstellar travel one need not create for a single trip such a huge cylinder with $D_{a}$ of the order of light years. It would suffice to surround the pilot with a small bubble — called the Alcubierre bubble ${ }^{1}$ — with the diameter $D_{b} \ll D_{a}$ (see figure 1b), which would reduce the area of the 'domain wall' surrounding the pilot — or, rather, of its outer surface - by $10^{32}$ (for $D_{a} \sim 100 \mathrm{ly}$ and $D_{b} \sim 100 \mathrm{~m}$ ).

The null cones in $Z$ (or in $Z_{b}$ see figure 1c) can be tilted so much (with the metric remaining flat) that a future-directed null vector is directed in the sense of decreasing $t$ [2] (which, being again a coordinate effect, has no direct connection with causality — the spacetime is globally hyperbolic). The difference between such a shortcut (called the Krasnikov tube [3]) and the Alcubierre bubble is of no significance for problems discussed in this paper [it becomes crucial in situations (see below), when one have to consider round trips].

Remark. Creation of a shortcut in itself requires some time. Moreover, under some mild assumptions about the properties of the 'building materials' (the absence of tachyons, essentially) this time is too large [2] in the following sense. If a $D_{a}=100$ light years, then a shortcut that would allow one to reach the star in $1 \mathrm{yr}$, cannot be built in less than $99 \mathrm{yr}$. So, a created, artificial shortcut (in contrast to a found, natural one, such as a relic wormhole, say) is a useful means of interstellar travel only when the large time taken by the first trip is

\footnotetext{
1 The metric originally proposed by Alcubierre [1] is a little different (in particular, the null cones also tilt inside the bubble, not just open), but the principle of operation is the same.
} 
not important $[2,4]$. This may be an exploratory expedition, (when only the time of return matters), or a regular space service between two stars.

It seems interesting from both academic and practical points of view to find out whether something like a shortcut can be found in nature or manufactured by an advanced civilization. Referring to an advanced civilization I mean that we are not concerned with 'technical details' such as: how to create a wormhole, how to penetrate a domain wall, etc. At this stage of research almost any spacetime may be acknowledged as being possible unless it clearly contradicts some fundamental laws or observations. It appears, however, that even by such liberal criteria the existence of shortcuts is questionable. As was shown in [3] maintaining an Alcubierre bubble $100 \mathrm{~m}$ in diameter involves energies of the order of $\sim 10^{67} \mathrm{~g} \approx 10^{34} M_{\odot}$. A similar result was obtained in [5] for the Krasnikov tube and, as we argue below, can be, analogously obtained for a traversable wormhole as well. Such a figure looks absolutely discouraging even with regard to an extremely advanced civilization and can be viewed as a prohibition of shortcuts.

The goal of this paper is to show that it is not impossible to get around this prohibition. This will be shown in section III after a brief discussion — in section II — of the origin and meaning of that awesome figure.

\section{RESTRICTIONS ON SHORTCUTS}

\section{A. Superluminal travel and the weak energy condition}

The root of the problems involved in creating a shortcut lies in the fact that, as was shown in [6], [1], and [3] for wormholes, Alcubierre's bubble, and Krasnikov's tube, respectively, the Weak energy condition (WEC)

$$
G_{\mu \nu} t^{\mu} t^{\nu} \geqslant 0 \quad \forall \text { timelike } \boldsymbol{t}
$$

must break down in some regions of these spacetimes. When the classical Einstein equations are adopted the violation of WEC implies that for some observers the energy density $T_{00}$ in those regions is negative, which is forbidden.

That a shortcut requires negative energy is hardly surprising — if the energy density were non-negative everywhere one would expect the total mass of the shortcut to be positive, while in fact it is zero by definition. Hence an important question arises: Is the necessity of 
negative energy densities something inherent in superluminal travel, or is it just an artifact of our approach, in which the spacetime outside some region is required to be strictly flat $^{2}$ and thus the gravitational fields of stars, nebulae, and other potential sources of the energy needed for the trip are neglected. It is instructive to compare the notion of a shortcut with its alternatives.

Consider a group of runners. The starting line and the finish line taken, respectively, at the moments, when the race begins and when the first runner finishes, are spacelike geodesics. We recognize a runner as the fastest if he or she is the only one whose world line intersects both these geodesics. Olum proposed [8] to use the same criterion to distinguish a 'fastest' null geodesic, which he calls superluminal (see also [9]). More specifically a null geodesic $\gamma$ connecting points $p$ and $q$ is called superluminal (in the (2+1)-dimensional case, which is easier to visualize) only if there are geodesics $\lambda_{p} \ni p$ and $\lambda_{q} \ni q$ such that of all their points only $p$ and $q$ are causally connected. And it is proved in [8] that if the generic condition holds on $\gamma$, then WEC does not.

The advantage of Olum's definition (or, rather, part of the definition, since only a necessary condition is formulated) is that one does not need compare objects and quantities belonging to different spacetimes. On the other hand, it excludes some paths which (on the same intuitive grounds) one might want to consider as superluminal. Suppose, for example, that $\lambda_{p}$ is the line $t=0, x=-2 c$ in a shortcut $M$ (the coordinates are pulled back by $\varkappa$ from the Minkowski space) and that the photons emitted from this line move with a constant $y$ and meet the plane $x=2 c$ in points $t=f(y)$. Now, according to Olum, if $f$ has a strict minimum (in $y_{0}$, say), it qualifies the geodesic emitted in $\lambda_{p}\left(y_{0}\right)$ as being superluminal, but not if the minimum is weak (which, for example, is the case with a 'portal' (see subsection III C 1), or an Alcubierre bubble if its front wall is flatten).

A different approach is proposed in [2] (see also [10]). The travel time $T_{\mathrm{R}}$ in the real world $M_{\mathrm{R}}$ - with all its shortcuts (if they are present there) and with (possibly non-trivial) interaction between the spaceship and its environment - is again compared with the travel time $T_{\mathrm{F}}$ in some fictitious spacetime $M_{\mathrm{F}}$. This time, however, $M_{\mathrm{F}}$ is not the Minkowski space, but the spacetime that would have formed if the trip had not been undertaken. As

\footnotetext{
${ }^{2}$ Note in this connection that, unfortunately, it is hard (if possible at all) to define reasonably a "nearly flat' spacetime. This is because, in contrast to the Riemannian case, there is no set of scalars such that their vanishing would guarantee that a spacetime is flat [7].
} 
an example (for the rigorous definition and some discussion see [2])) consider a spherically symmetric spacetime which is empty outside a cylinder $N=B_{r_{o}} \times \mathbb{L}^{1}$, where $B_{r_{o}}$ is a (3-dimensional) ball with the radius $r_{o}$. Let us interpret this spacetime as a model of a neighbourhood of a globular cluster. At $t=0$ an observer located in a point with $r=$ $r_{S}>r_{o}$ decides to explore a star located in the diametrically opposite point and considers the following two scenarios. First, he/she can send a photon, which would pass through the cluster, reflect (at $t=T_{D}$, say) from something near the desired star, pass through the cluster once again (in the opposite direction), and, finally, return to $r=r_{S}$. The other possibility is to send a spaceship instead of the photon. Being powerful enough, the spaceship on its first passage through the cluster would push the stars, blow them up, emit different (but all satisfying WEC) fields, etc. As a result the metric at $t=T_{D}$ in this second scenario differs from that in the first (though, if the spherical symmetry is preserved by the pilot, it may remain, say, Schwarzschild, outside $N$ ). So, it is not surprising that the back way - and thus the whole trip - takes different time $\left(T_{\mathrm{F}}\right.$ and $T_{\mathrm{R}}$, respectively) for the photon in the first scenario and for the spaceship in the second. And if $T_{\mathrm{R}}<T_{\mathrm{F}}$ such a trip deserves to be called superluminal. A superluminal, in this sense, journey does not require violation of WEC. In the appendix we prove this fact (though actually it is almost self-evident) by constructing a specific example.

The problem with this example is that $T_{\mathrm{R}}$ in it, though being less than $T_{\mathrm{F}}$, is still greater than $T_{\mathrm{M}}$, where $T_{\mathrm{M}}=4 r_{S}$ is the time required for the same trip in the Minkowski space (by the 'same' trip I mean the trip between the points with the same $t, r, \phi$, and $\theta$ coordinates; due to the spherical symmetry and staticity of $M_{\mathrm{F}}$ and $M_{\mathrm{R}}-N$ such a mapping on the Minkowski space is more or less meaningful, see [9] though). So one can interpret $T_{\mathrm{F}}-T_{\mathrm{R}}$ to be not so much a gain in travel time as some compensation of the time delay $T_{\mathrm{F}}-T_{\mathrm{M}}$ experienced by a traveler in $M_{\mathrm{F}}$ and caused by the variation of $M_{\mathrm{F}}$ from the Minkowski space.

\section{B. The 'total negative energy' $E_{\text {tot }}^{-}$}

Since the classical matter (alone) cannot sustain a shortcut we turn our attention to the quantum effects — described within the semiclassical approximation [11] — and, corre- 
spondingly, cast the Einstein equations into the form

$$
G_{\mu \nu}=8 \pi T_{\mu \nu}^{\mathrm{C}}+8 \pi\left\langle T_{\mu \nu}\right\rangle
$$

Here $T_{\mu \nu}^{\mathrm{C}}$ is the contribution of the 'classical matter', that is the matter for which quantum effects can be neglected. $T_{\mu \nu}^{\mathrm{C}}$ is supposed to obey the Weak energy condition, but is otherwise arbitrary. As for the second term, it is the (renormalized) expectation value of the stressenergy tensor of quantum fields involved. As is well known $\left\langle T_{\mu \nu}\right\rangle$ may violate WEC and so the necessity of negative energy densities does not by itself exclude the shortcuts. It was found, however (see [12] for a review), that such violations are not arbitrary, but may be subject to a restriction called the quantum inequality (QI). The remainder of this subsection is a (very sketchy) review of how QI in its turn imposes restrictions on shortcuts.

Consider the free electro-magnetic or scalar (massless, minimally-coupled) field in the Minkowski space. Let $\bar{\varrho}_{\chi}$ be its energy density averaged with a weighting function $\chi$ over a timelike geodesic $\gamma(\tau)$

$$
\bar{\varrho}_{\chi}\left(\tau_{0}, \Delta\right) \equiv \int_{-\infty}^{\infty}\left\langle T_{\mu \nu} u^{\mu} u^{\nu}\right\rangle \chi\left(\tau_{0}, \Delta ; \tau\right) \mathrm{d} \tau
$$

( $\tau$ is the proper time on $\gamma$ and $\boldsymbol{u} \equiv \partial_{\tau}$ ). It is assumed that $\chi(\tau)$ is smooth, its integral is unity, and its support lies in $\left(\tau_{0}-\Delta / 2, \tau_{0}+\Delta / 2\right)$, so that, when $\Delta$ is small,

$$
\bar{\varrho}_{\chi}\left(\tau_{0}, \Delta\right) \approx \varrho\left(\tau_{0}\right)
$$

where $\varrho \equiv\left\langle T_{\mu \nu} u^{\mu} u^{\nu}\right\rangle$ is the energy density of the field as measured by an observer with the world line $\gamma$. This equality allows one to estimate the energy density, because $\bar{\varrho}_{\chi}$ obeys the following 'quantum inequality' [13]

$$
\bar{\varrho}_{\chi} \geqslant-A \Delta^{-4}
$$

where $A$ is a positive constant of order of unity (from now on I freely omit insignificant constants like $A, 8 \pi$, etc.).

Assume now (cf. $[3,5])$ that (4) is valid also in curved spacetime if $\Delta$ is sufficiently small, or, more specifically [14], if the following holds (actually, one more inequality is implied, see the next section)

$$
\Delta \lesssim \ell \equiv\left(\max \left|R_{\alpha \beta \gamma \delta}\right|\right)^{-1 / 2}
$$


where the components of the Riemann tensor are found in the observer's proper frame. The condition (5) is supposed to guarantee that the relevant segment of $\gamma$ lies in a region so small that it can be regarded as 'approximately Minkowskian'. The quantum inequality (4) [allowing for (3) and (5)] relates the curvature in a point with the energy density in this point. It restricts, loosely speaking, the amount of 'exotic matter' (i. e. matter violating the Weak energy condition [6]) that may be produced by the curvature of spacetime. But (almost) the same quantities are related also by the Einstein equations $G_{00}=8 \pi \varrho$. Ignoring in our rough consideration the possible difference between the scales given by the components of the Einstein and of the Riemann tensors, we can combine the two relations to obtain

$$
\varrho \sim \bar{\varrho}_{\chi} \geqslant-\Delta^{-4} \sim-\ell^{-4} \sim-\varrho^{2}
$$

which, when $\varrho$ is negative, gives

$$
|\varrho| \gtrsim 1
$$

It is this Planck-scale energy density that gives rise to the prohibitive figures cited in the introduction (recall that $1 \approx 5 \times 10^{93} \mathrm{~g} / \mathrm{cm}^{3}$ ). Indeed, let us estimate the total amount of negative energy $E_{\text {tot }}^{-}$required for maintenance of a shortcut. We define it as follows

$$
E_{\text {tot }}^{-} \equiv \int_{\Xi}|\varrho| \mathrm{d}^{3} x
$$

Here we have chosen a spacelike surface $\mathcal{S}$ in $M$ and denoted by $\Xi \subset \mathcal{S}$ the region within it in which WEC is violated.

$E_{\text {tot }}^{-}$is approximately equal to $-\varrho V_{\Xi}$, where $V_{\Xi}$ is the volume of $\Xi$. In both Alcubierre and Krasnikov spaces $\Xi$ is a spherical layer ('domain wall') surrounding the domain $\mathcal{D}$ of the 'false' flat metric. The volume of the domain wall can be estimated as $V_{\Xi} \gtrsim S_{\mathrm{i}} \delta$, where $S_{\mathrm{i}}$ is the area of its inner surface (recall that as discussed in the introduction, the area of the outer surface is much greater, even though $\delta$ is small). $\mathcal{D}$ must be at least $\sim 1 \mathrm{~m}$ in diameter — which means that $S_{\mathrm{i}} \gtrsim 10^{70}$ — to accommodate a human. For a spherically symmetric wormhole, $\Xi$ is essentially the throat of the wormhole, that is also a spherical layer with the radius $\gtrsim 1 \mathrm{~m}$ if a human being is supposed to pass through it. So, even if the thickness of the layer $\delta \sim l_{\mathrm{Pl}}$, one might conclude that it would take at least

$$
E_{\text {tot }}^{-} \sim\left|\varrho S_{\mathrm{i}} \delta\right| \approx 10^{32} M_{\odot}
$$

of exotic matter to support a shortcut. Such a huge value presumably indicates the "unphysical nature' [5] of shortcuts. 
Example. Consider a Morris-Thorne wormhole

$$
\mathrm{d} s^{2}=-\mathrm{d} t^{2}+\mathrm{d} l^{2}+r(l)^{2}\left(\mathrm{~d} \theta^{2}+\sin ^{2} \theta \mathrm{d} \phi^{2}\right),
$$

where $r(l)$ is a smooth even function with a single minimum $r(0)=r_{0}$. At positive $l$ (i. e. at $r>r_{0}$ ) we can choose $r$ to be a coordinate and rewrite the metric in the following form

$$
\mathrm{d} s^{2}=-\mathrm{d} t^{2}+\frac{r}{r-b(r)} \mathrm{d} r^{2}+r^{2}\left(\mathrm{~d} \theta^{2}+\sin ^{2} \theta \mathrm{d} \phi^{2}\right),
$$

where $b(r)$ can be, if desired, expressed in terms of $r^{\prime}(l)$. In the special case in which ${ }^{3}$

$$
b(r)=\left\{\begin{array}{ll}
r_{0}\left[1-\left(r-r_{0}\right) / \delta\right]^{2}, & \text { at } r \in\left(r_{0}, r_{0}+\delta\right] \\
0 & \text { at } r \geqslant r_{0}+\delta
\end{array}, \quad \delta \ll r_{0}\right.
$$

the wormhole $(11,12)$ is called 'absurdly benign' [6]. The spacetime is flat except for a spherical $\delta$ thick layer $\Xi$ in which $b \neq 0$. The energy density in this layer is

$$
G_{\hat{t} \hat{t}} \sim-\left(\delta r_{0}\right)^{-1}
$$

and it is easy to see that (cf. endnote 25 of [3])

$$
E_{\text {tot }}^{-} \sim G_{\hat{t} \hat{t}} V_{\Xi} \sim r_{0} \approx 10^{-3} M_{\odot}\left(\frac{r_{0}}{1 \mathrm{~m}}\right),
$$

which looks of course far more attractive than (9). Let us see, however, what restrictions on the parameters of the wormhole are imposed by QI. The maximal component of the Riemann tensor in an orthonormal frame of a static observer located in the throat (i. e. near $\left.r=r_{0}\right)$ is

$$
\left|R_{\hat{r} \hat{\phi} \hat{\phi} \hat{\phi}}\right| \approx \frac{1}{\delta r_{0}}
$$

Correspondingly, QI $(4,5)$ requires that

$$
\bar{\varrho}_{\chi} \gtrsim-\left(\delta r_{0}\right)^{-2}
$$

(as we shall see in a moment, this is a vastly more restrictive condition than $\bar{\varrho}_{\chi} \gtrsim-\delta^{-4}$, which is considered in [14]). $\varrho$ does not depend on $\tau$ for a so chosen observer and hence

$$
\bar{\varrho}_{\chi}=\varrho=\left.G_{\hat{t} \hat{t}}\right|_{r=r_{0}} \sim-\left(\delta r_{0}\right)^{-1}
$$

\footnotetext{
${ }^{3}$ It is understood that actually $b(r)$ is smoothed near $r=r_{0}+\delta$ in a manner that does not affect the following considerations.
} 
Comparing this with the preceding inequality we find that $\varrho \lesssim-1$, as expected, and $\delta \lesssim$ $1 / r_{0}$. So, the frugality of the wormhole owes just to the fact that the thickness $\delta$ of the curved region is not of the order of $l_{\mathrm{Pl}}$ (as we took in deriving $(9)$ ), but of the order of $10^{-35} l_{\mathrm{Pl}}$, which is a value, of course, that makes the wormhole much more absurd than benign.

\section{WAYS OUT}

The analysis of the previous section leading to the restrictions (7) and (9) and to their interpretation is quite rough, of course. To some extent it can be refined, see $[3,5,14]$, but, as we discuss in this section, significant loopholes remain.

In the search for realistic shortcuts an obvious line of attack would be to look for situations in which the quantum inequality (4) does not hold. Note in this connection that for non-Minkowskian spacetimes (4) has never been proved ${ }^{4}$, though some arguments in its substantiation were brought forward in [14]. Moreover, in their recent paper [17] Olum and Graham showed that a system of two interacting scalar fields may violate (4). (It is especially interesting that the violation takes place near a domain wall. One might speculate on this ground that perhaps the similarity of the shortcuts considered in the introduction with the domains of false vacuum has far-reaching consequences.) Thus the inequality (4) is, at least, non-universal.

We shall, however, explore another possibility. In what follows we demonstrate that some (perhaps most) shortcuts are not excluded even if QI does hold.

\section{A. $E_{\text {tot }}^{-}$indeterminate}

The derivation of (7) rests heavily on the assumption that (at least in $\Xi$ ) the components of the Riemann and the Einstein tensors are roughly of the same order: in the key relation

$$
\ell^{-4} \sim \rho^{2}
$$

\footnotetext{
${ }^{4}$ The only exception, to my knowledge, is the two-dimensional conformally trivial case [15]. There are also 'difference inequalities' (see, e. g., [16]), but, though resembling (4) in appearance, they differ from it fundamentally (they restrict a part $\left\langle: T_{\mu \nu}:\right\rangle$ of the full expectation value of the stress-energy tensor $\left.\left\langle T_{\mu \nu}\right\rangle\right)$ and therefore cannot be (directly) used to derive restrictions like (9).
} 
(see the end of the chain (6)) the left hand side is determined by the former and the right hand side by the latter. Physically this assumption means that the case is considered in which the exotic matter is generated mostly by the curvature produced by this same matter (for a moment we ignore other possible mechanisms of generating exotic matter, such as mirrors). But the vacuum polarization in a point is determined (among other things) by the Weyl tensor $C_{\alpha \beta \rho \sigma}$ and not exclusively by the Ricci tensor $R_{\alpha \beta}$. In particular, the anomalous trace of the conformal scalar field is given [11] by

$$
\left\langle T_{\mu}^{\mu}\right\rangle=\frac{1}{2880 \pi^{2}}\left(C_{\alpha \beta \rho \sigma} C^{\alpha \beta \rho \sigma}+R_{\alpha \beta} R^{\alpha \beta}-\frac{1}{3} R^{2}+\square R\right)
$$

So, one expects (13) to be true only when

$$
\max \left|C_{\alpha \beta \gamma \delta}\right| / \max \left|R_{\alpha \beta}\right| \lesssim 1
$$

a condition that breaks down more often than not. For example, in any curved and empty region (e. g. in the vicinity of any star)

$$
\max \left|C_{\alpha \beta \gamma \delta}\right| / \max \left|R_{\alpha \beta}\right|=\infty
$$

And relaxing the condition (14) (and hence (13)) one immediately removes the restriction (7) and invalidates (9).

Another situation in which the quantum inequality does not imply (7) and (9) concerns wormholes. The point is that QI presumably holds for a sufficiently small $\Delta$ because 'a curved spacetime appears flat if restricted to a sufficiently small region' [14] and hence the condition (5). A spacetime, however, may differ from the Minkowski space in global properties. As is well known, $\varrho$ is negative and constant (in contradiction to (4)) even in a flat spacetime, if the latter is warped into a cylinder (the Casimir effect). To handle such situations it was proposed in [14] to supplement (5) with a requirement that $\Delta$ be much less than 'the proper distance from the point $\gamma\left(\tau_{0}\right)$ to the boundary of the spacetime'. In particular, if in a certain direction a condition of periodicity — of length $L$ — is imposed on a field (that is, a field on a cylinder, or, say, between mirrors is considered), then it is required that at least ${ }^{5}$

$$
\Delta \lesssim L
$$

\footnotetext{
${ }^{5}$ In [14] even a more restrictive requirement $\Delta \lesssim L / \gamma$ is imposed, where $\gamma \geqslant 1$.
} 
This condition cannot be significantly weakened since for the massless scalar field and a static observer we have [11] in the Casimir case

$$
\varrho=-\pi^{2} L^{-4} / 1440
$$

To see the implications of (15) for shortcuts, consider a spacetime $M_{T}$ obtained by the following procedure (see figure 2a). First, two thin vertical, i. e. parallel to the $t$-axis, cylin-

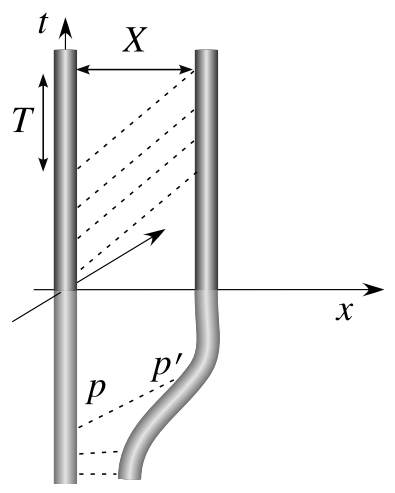

(a)

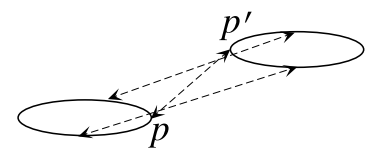

(b)

FIG. 2: (a) The throat of the wormhole is required to be constant. So if $p$ is identified with $p^{\prime}$, then the other pairs of identified points satisfy $\tau=\tau^{\prime}$, where $\tau^{(\prime)}$ is the proper time measured along the cylinder from $p^{(\prime)}$ to the corresponding point. Accordingly, $T$ changes if a mouth moves. (b) The dashed lines show how the identification must be performed.

ders are removed from the Minkowski space. Then some (close) vicinities of the boundaries of the holes are appropriately curved (so that the resulting spacetime be smooth). Finally, the boundaries are identified according to the following rule: each section $t=t_{0}$ of the left cylinder, which is a two dimensional sphere, is glued to the section $t=t_{0}+T$ of the right cylinder. The identification of the two spheres is performed so (see figure $2 \mathrm{~b}$ ) that the segment $\left(p p^{\prime}\right)$, which lies in the $(t, x)$ plane and connects the spheres, becomes a circle.

The spacetime $M_{T}$ describes a wormhole whose both mouths are at rest with respect to an inertial observer located in the Minkowskian part of the shortcut (i. e. in $U$, see the definition of the shortcut). Given such a wormhole it is relatively easy to impart a desired value of $T$ to it. All one needs is to move one of the mouths [18] without changing the geometry of the throat (and thus without spending much energy). 
Since shortcuts by definition must be globally hyperbolic we restrict ourselves to the spaces with $X>T$ (lest the spacetime contain closed causal curves with all the ensuing complications $[18,19])$. Among these, $M_{T}$ with $X \approx T$ are most interesting. On the one hand, such a wormhole may be an efficient shortcut: to make a distant journey one acquires a wormhole with both its mouths initially located near the Earth and takes one of them with him/her. Moving at a high speed the traveler reaches the destination in a short (proper) time $\Delta \tau$ (by this time $X$ becomes $\approx T$ ). The return trip is made through the wormhole and (neglecting the time spent on traversing the wormhole) the traveler returns to the Earth in $\Delta \tau$ (by the terrestrial clock) after the departure.

And, on the other hand, such $M_{T}$ are free from the restrictions (7,9) imposed by QI. Indeed, the section $y=z=0$ of $M_{T}$ is a cylinder with $L=\sqrt{X^{2}-T^{2}}$. So, whatever $\varrho$ is required by the Einstein equations to support the wormhole, it suffices to make $L$ sufficiently small (i. e. $X$ sufficiently close to $L$ ) and the quantum inequality, owing to (15), will be satisfied. Note that $L$, if desired, can be made arbitrarily small (e. g. $L=l_{\mathrm{Pl}}$ ) without any damage to the traversability of the wormhole: the radius of its throat and the distance $X$ between the mouths will remain macroscopic.

Moreover, by analogy with the Casimir effect (16), it is reasonable to assume that $\varrho$ in such a wormhole will be large $\left(\sim L^{-4}\right)$, which would relieve one of having to seek additional sources of exotic matter.

\section{B. $E_{\text {tot }}^{-}$large, but meaningless}

Suppose working within classical electrostatics we discover that for some field configuration the energy $E_{\Upsilon}$ of the field $\boldsymbol{E}(x)$ contained in a region $\Upsilon$ is unphysically large. Say,

$$
E_{\Upsilon}=\frac{1}{8 \pi} \int_{\Upsilon} E^{2}(x) \mathrm{d}^{3} x=10^{32} M_{\odot} .
$$

Should we conclude based on (17) alone that the configuration $\boldsymbol{E}(x)$ is unphysical? The answer is obviously negative. (17) is true, for example, when $\boldsymbol{E}(x)$ is just the field of a pointlike charge and $\Upsilon$ is the complement to a (small enough) ball $B_{r_{c}}=\left\{x: r(x)<r_{c}\right\}$ around the charge. The reason why such a respectable solution of the Maxwell equations involves such huge energies is trivial. $\boldsymbol{E}(x)$ is not the real value of the field in $x$, but only its value assuming that the effects lying beyond the model (classical electrostatics) 
are negligible, which is certainly not true when the main contribution comes from a transPlanckian region $r \sim r_{c}$. Of course the failure to predict the field, or the energy density, in $B_{r_{c}}$ does not compromise electrostatics. It only reminds us that the values of the field $\boldsymbol{E}$, or potential $\varphi$ in a point, are not physically meaningful but rather their averages $\overline{\boldsymbol{E}}, \bar{\varphi}$ are, defined, say, as

$$
\bar{\varphi}\left(x_{0}\right)=\frac{3}{4 \pi r_{l}^{3}} \int_{B_{r_{l}}} \varphi \mathrm{d}^{3} x,
$$

where $r_{l}$ is chosen so large that quantum fluctuations do not affect $\bar{\varphi}$. While our judgments about $\varphi\left(x_{0}\right)$, say, are valid only as far as it is possible to find $r_{l}$ such that $\bar{\varphi}\left(x_{0}\right)-\varphi\left(x_{0}\right)$ is negligible.

The same considerations fully apply to semiclassical gravity. It is not supposed to predict (correctly) the values of the relevant quantities (such as $g_{\mu \nu},\left\langle T_{\mu \nu}\right\rangle_{0}$, etc.) unless the contribution of quantum gravitational effects can be neglected. Which means that the enormous values of $E_{\text {tot }}^{-}$may testify not to the fact that the involved energies are unphysically large, but just that $E_{\text {tot }}^{-}$is found incorrectly (that is with illegal neglect of quantum corrections).

It is worth noting that metrics of this kind - on the one hand they are solutions of semiclassical equations and on the other hand they violate WEC in regions $\Xi$ so small that one cannot properly assess $E_{\text {tot }}^{-}$by means of semiclassical gravity - arise naturally in constructing macroscopic self-maintained wormholes. Consider, for example, a metric [20]

$$
\mathrm{d} s^{2}=\Omega^{2}(\xi)\left[-\mathrm{d} \tau^{2}+\mathrm{d} \xi^{2}+K^{2}(\xi)\left(\mathrm{d} \theta^{2}+\sin ^{2} \theta \mathrm{d} \phi^{2}\right)\right],
$$

where $K$ and $\Omega$ are positive even functions, such that $K(\xi)$ and $e^{-\xi / \xi_{0}} \Omega(\xi)$ tend to non-zero constants at large $\xi$. This metric is increasingly flat, (that is the gravitational field falls with $|\xi|$, though it does not vanish completely and so (19) is not a shortcut), which means that it describes a wormhole ${ }^{6}$. No restrictions are imposed on $\min \Omega K$, which is the radius of the wormhole's throat (again the actual radius, i. e. the quantity that matters, when one decides whether a wormhole can be traversed, is of course $\min \bar{\Omega} \bar{K})$. In particular, it may be macroscopic qualifying thus the wormhole as traversable. The importance of such wormholes lies in the fact that some of them are solutions of the Einstein equations (2) with

\footnotetext{
${ }^{6}$ Though the metric (19) is spherically symmetric and static it is not of the Morris-Thorne type [6]. So it may seem somewhat surprising that it is, nevertheless, a wormhole. It becomes obvious, however, in appropriately chosen coordinates.
} 
$\left\langle T_{\mu \nu}\right\rangle$ being the vacuum expectation value of the stress-energy tensor of a realistic (i. e. electromagnetic, neutrino, or massless scalar) field. That is they describe the result of the following scenario: at some moment — say, at the Big Bang — a wormhole comes into existence. The non-flatness of the spacetime near the throat polarizes the vacuum and the term $\left\langle T_{00}\right\rangle$, if it is negative and large enough, supports the wormhole and prevents it from collapsing. If it fails, the wormhole begins to collapse and, correspondingly, the vacuum polarization increases. The process goes on until the equilibrium is (hopefully) found and the wormhole acquires the shape (19). How much energy this process requires is, of course, anybody's guess.

Remarkably, such solutions have $\Omega$ oscillating in the throat (i. e. at $\xi \sim 0$ ). To leading order it may have the form [20]

$$
\Omega_{0} \exp \left[\epsilon \sin \left(\xi / \xi_{0}\right)\right]
$$

with $\epsilon, \xi_{0} \ll 1$, so the oscillations have exceedingly small magnitude and wavelength. The energy density of the field (if found according to the semiclassical rules) changes its sign with the sine and thus $\Xi$ is a set of concentric spherical layers, each $\pi \xi_{0} \ll 1$ thick. Therefore, as discussed above, one can hardly expect that $E_{\text {tot }}^{-}$defined by (8) is something measurable. Note, however, that it is quite different with the components of the metric: we can take $r_{l}$ in (18) to be, say, of the order of $100 l_{\mathrm{Pl}}$ and check that $\bar{\Omega} \approx \Omega$. Thus the model contains quantities of two types — some are physically meaningful and trustworthy as long as we trust semiclassical gravity (these, for example, are $\bar{\Omega}, \bar{K}$, etc.), and the others are purely auxiliary, devoid of any specific physical meaning $\left(\Omega, E_{\text {tot }}^{-}\right.$, etc.).

\section{C. $E_{\text {tot }}^{-}$small}

\section{1. 'Portal'}

In this section we show that in the case of a wormhole just by abandoning the spherical symmetry it is possible to reduce $V_{\Xi}$ (and thus $E_{\text {tot }}^{-}$) drastically - by 35 orders in this case. The proposed wormhole ${ }^{7}$ also has another advantage: a traveler taking the short cut moves all the time in a flat region and need not plunge into the Planck-density matter (7).

\footnotetext{
7 which is, in fact, a globally hyperbolic analog of the 'dihedral' wormhole [21].
} 
Consider a spacetime $W$

$$
\mathrm{d} s^{2}=-\mathrm{d} t^{2}+4\left(\varepsilon^{2}(\eta)+\eta^{2}\right)\left(\mathrm{d} \eta^{2}+\eta^{2} \mathrm{~d} \psi^{2}\right)+\rho^{2} \mathrm{~d} \phi^{2},
$$

where $\varepsilon$ is a smooth even function whose support is the region $\mathcal{E} \equiv\left\{\eta<\eta_{\varepsilon}\right\}$;

$$
\rho \equiv \rho_{0}-\eta^{2} \cos 2 \psi
$$

and $\rho_{0}, \eta_{\varepsilon}$ are positive constants $\rho_{0} \gg \eta_{\varepsilon}^{2}$. As usual, it is understood that $\eta, \rho \geqslant 0, \phi=\phi+2 \pi$, $\psi=\psi+2 \pi$ and that the points with $\eta=0$ differing only by $\psi$ are identified, as are the points with $\rho=0$ differing only by $\phi$.

To visualize the structure of $W$ and to check that the singularity in $\rho=0$ is coordinate, consider, first, the region $W-\mathcal{E}$. Defining $z \equiv \eta^{2} \sin 2 \psi$ we isometrically map $W-\mathcal{E}$ on a spacetime $U$, which is the Minkowski space

$$
\mathrm{d} s^{2}=-\mathrm{d} t^{2}+\mathrm{d} z^{2}+\mathrm{d} \rho^{2}+\rho^{2} \mathrm{~d} \phi^{2},
$$

from which at each $t$ a solid torus $\Xi=\left\{\left(\rho-\rho_{0}\right)^{2}+z^{2}<\eta_{\varepsilon}^{4}\right\}$ is removed:

$$
(W-\mathcal{E}) \stackrel{\zeta}{\rightarrow} U \equiv \mathbb{L}^{4}-\Xi \times \mathbb{L}^{1} .
$$

Locally $\zeta$ is an isometry. At the same time it sends each pair of points $(t, \phi, \eta, \psi),(t, \phi, \eta, \psi+$ $\pi)$ to a single point $(t, \phi, \rho, z) \in U$ as shown in figure 3a. So, $W-\mathcal{E}$ is the two-fold covering of $U$. To put it another way, $W-\mathcal{E}$ can be constructed by taking two copies - let us call them $U_{1}$ and $U_{2}$ - of $U$, cutting each of them along the disk $\left(z=0, \rho<\rho_{0}\right)$, and gluing the right bank of either cut to left bank of the other.

$W$ is a wormhole connecting two 'different spacetimes' $U_{1}$ and $U_{2}$. Now we shall construct from it a shortcut $P$, i. e., in this instance, a wormhole that connects remote parts of the 'same' spacetime. To this end we remove the region $z>D(D$ is a constant greater than $\eta_{\varepsilon}^{2}$ ) from $U_{1}$ and the region $z<-D$ from $U_{2} . \quad P$ is obtained by gluing together the two boundaries $-z=D$ in $U_{1}$ and $z=-D$ in $U_{2}$ (note that the surgery takes place in the Minkowski part of $W$ and thus, obviously, does not give rise to any singularities).

Alternatively construction of $P$ can be described in terms of $\eta$ and $\psi$. First, remove from the plane $(\eta, \psi)$ the regions $\rho<0$ (these are the interiors of the upper and the lower hyperbolae in figure 3a). If we rotate now the remaining part of the plane with respect to 


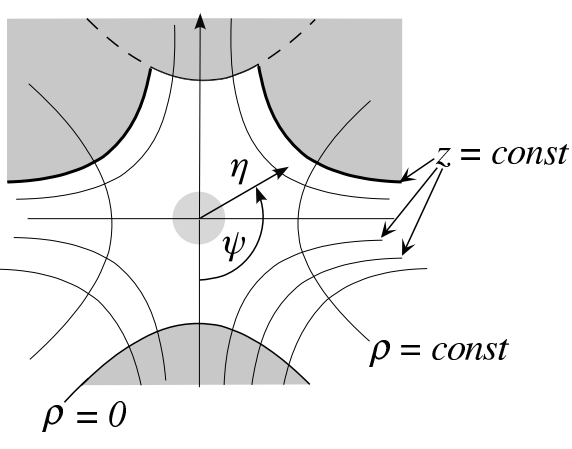

(a)

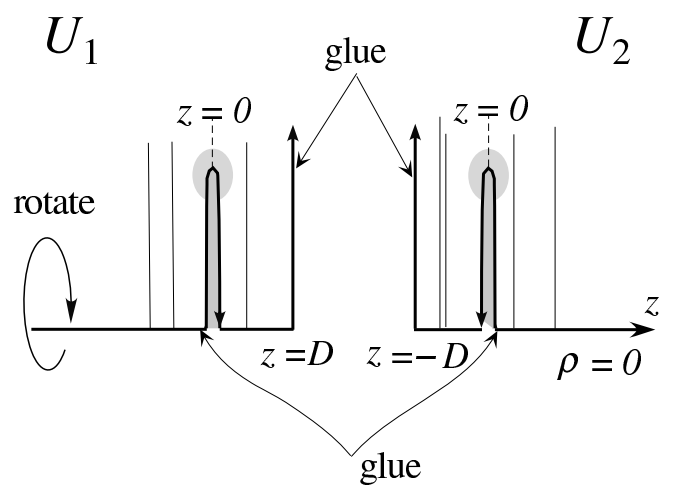

(b)

FIG. 3: A section $\phi=t=0$ of the spacetime $P$. The dark gray regions do not belong to $P$. The thick (segments of) hyperbolae are identified. $\zeta$ maps the left (right) half of (a) to the left (right) half of (b). The spacetime is flat except in the light gray regions.

the $z$-axis, we would obtain $W$. And $P$ is obtained if before the rotation we remove the regions

$$
\sin \psi<0, \quad \eta^{2} \sin 2 \psi>D \quad \text { and } \quad \sin \psi>0, \quad \eta^{2} \sin 2 \psi<-D
$$

(i. e. the interiors of the left and the right hyperbolae) and identify their boundaries.

$P$ is a globally hyperbolic and static wormhole. Its spacelike section $P_{(3)}$ has the structure shown in figure 4 . Outside some compact region $P_{(3)}$ is just the Euclidean space. And inside

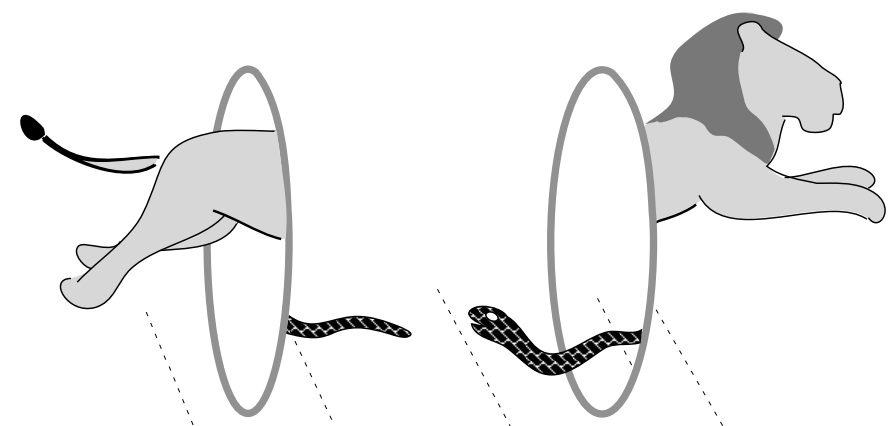

FIG. 4: The 'distance between the hoops' (defined, for example, as the length of the snake) is $2 D$. The radius of each is $\rho_{0}$ and the thickness is $\sim \eta_{\varepsilon}^{2}$. 
this region the space is flat too, except for two hoops (which, in fact, are a single hoop). A traveler passing through one of the hoops instantly finds himself emerging from the other one (remarkably, throughout the whole journey the spacetime around the traveler remains empty and flat).

To estimate the required $E_{\text {tot }}^{-}$let us choose the following $\varepsilon$

$$
\varepsilon^{2}=\frac{1}{4}\left(\eta_{\varepsilon}+\eta^{2} / \eta_{\varepsilon}\right)^{2}-\eta^{2} \quad \text { at } \eta \leqslant \eta_{\varepsilon}, \quad \varepsilon=0 \quad \text { at } \eta \geqslant \eta_{\varepsilon},
$$

so that the metric and its first derivatives are continuous in $\eta_{\varepsilon}$ (cf. footnote 3 ). With such a choice of $\varepsilon$

$$
G_{\hat{t} \hat{t}}=-\frac{4 \eta_{\varepsilon}^{4}}{\left(\eta^{2}+\eta_{\varepsilon}^{2}\right)^{4}}, \quad V_{\Xi}=\frac{14}{3} \pi^{2} \rho_{0} \eta_{\varepsilon}^{4},
$$

whence $E_{\text {tot }}^{-} \sim \rho_{0}$. So, to support a human-sized wormhole of this type it would suffice $E_{\text {tot }}^{-} \approx 10^{-2} M_{\odot}$ of exotic matter. This trifling, in comparison with (9), energy is about the energy of a supernova. QI, if it holds, does not change this estimate in any way. As with the absurdly benign wormhole (see section II B) it only requires that the hoops be thin (but not that thin in this case): $\eta_{\varepsilon} \sim 1$.

\section{Van Den Broeck's trick}

In fact, $E_{\text {tot }}^{-}$can be reduced further by tens of orders. Consider the metric (10), where this time $l \geqslant-l_{0}, r\left(-l_{0}\right)=0$ (so, the spacetime is $\mathbb{R}^{4}$ and not a wormhole), and $r(l)$ satisfies the following conditions

$$
\left.r\right|_{l \neq-l_{0}}>0,\left.\quad r^{\prime}\right|_{|l|>l_{2}}=1, \quad\left|r^{\prime}\right| \leqslant 1, \quad r^{\prime \prime}>0 \Leftrightarrow|l|<l_{1},
$$

where $l_{i}$ are positive constants: $l_{2}<l_{1}<l_{0}$ (see figure 5a). The spacetime $(10,20)$ (or, rather, its section $t, \theta=0$ ) is depicted in figure 5b. The Einstein tensor for the metric (10) can be easily found (see, e. g., (14.52) of [22]):

$$
G_{\hat{t} \hat{t}}=\frac{1-r^{\prime 2}-2 r r^{\prime \prime}}{r^{2}}, \quad G_{\hat{t} \hat{t}}+G_{\hat{r} \hat{r}}=-\frac{2 r^{\prime \prime}}{r}, \quad G_{\hat{t} \hat{t}}+G_{\hat{\nu} \hat{\nu}}=\frac{1-r^{\prime 2}-r r^{\prime \prime}}{r^{2}},
$$

where $\nu=\phi, \theta$. It follows that WEC breaks down (only) in the spherical layer $l \in\left(-l_{1}, l_{1}\right)$.

Let us choose ${ }^{8} r=\frac{1}{2 l_{1}} l^{2}+\frac{l_{1}}{2}$ on the interval $l \in\left(-l_{1}, l_{1}\right)$. Then $G_{\hat{t} \hat{t}} \geqslant-8 l_{1}^{2}$ and, since

\footnotetext{
${ }^{8}$ We take $r$ different from that proposed in the original paper [23], because the latter, first, leads to a much (by $\sim 10^{36}$ ) greater amount of exotic matter and, second, violates the quantum inequality (e. g. in $w=0.98)$.
} 


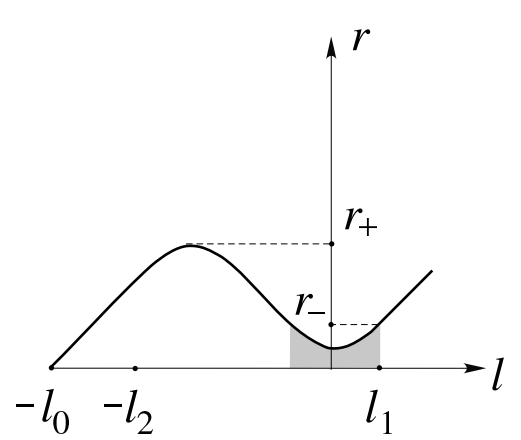

(a)

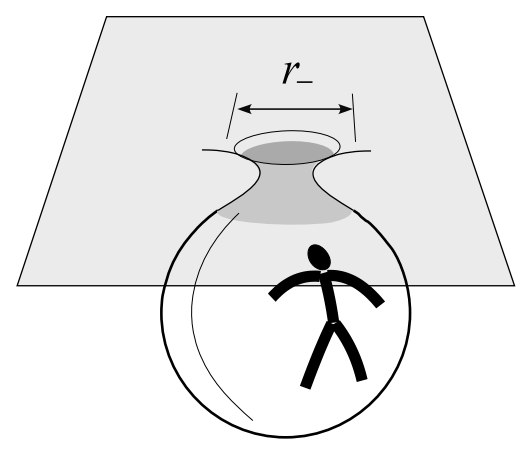

(b)

FIG. 5: With just $\sim-10^{-5}$ g of exotic matter one can make a Planck-size (in the view of an outside observer) capsule be arbitrarily roomy.

the volume of the layer is $V_{\Xi} \sim 4 \pi^{2} l_{1}^{3}$, the total amount of exotic matter is $\sim 10^{2} l_{1}$. We can (and, if (7) holds, we must) take $l_{1} \sim 1$ and hence it takes only $\sim-10^{-3}$ g of exotic matter to support the shape of the spacetime in discussion.

The pocket $(10,20)$ itself is not a shortcut, but an effective means for reducing the amount of negative energy required for a regular shortcut. The point is that, while the whole structure is enclosed within a sphere of the radius $r_{-}$from the point of view of an outside observer (see figure 5b), $r_{+}$can be chosen large enough to accommodate a passenger, or cargo. In other words, to transport a macroscopic passenger we can use now, say, a portal described in the previous subsection with $\rho_{0}=10 r_{-} \sim 10 l_{\mathrm{Pl}}$, which would require only $\sim-10^{-4} \mathrm{~g}$ of exotic matter (in addition to $-10^{-3} \mathrm{~g}$ spent on sustaining the pocket).

\section{Acknowledgements}

I am grateful to L. H. Ford and M. J. Pfenning for their critical comments and to C. J. Fewster for his remarks on the 'difference quantum inequality'. 


\section{APPENDIX}

In this section we construct a spherically symmetric spacetime $M$ with the following properties (cf. subsection II A):

1. $M$ is Schwarzschild outside a cylinder $N=B_{r_{o}} \times \mathbb{L}^{1}$;

2. The Weak energy condition holds in the whole $M$;

3. The minimal time taken by a trip through $B_{r_{o}}$ from a point with $r>r_{o}$ to the diametrically opposite point decreases with time.

First, let us introduce the following two functions

$$
\begin{gathered}
m(r) \equiv m_{0} r^{-1 / 3} \exp \int_{r_{h}}^{r} \frac{\vartheta(x) \mathrm{d} x}{3 x}, \\
\epsilon(r) \equiv \int_{r_{o}}^{r}(r-x) \frac{x^{2}\left(m^{\prime}(x) x^{-2}\right)^{\prime}}{x-2 m(x)} \varphi(x) \mathrm{d} x,
\end{gathered}
$$

where $m_{0}$, and $r_{h}<r_{o}$ are some positive constants. $\vartheta, \varphi$ are smooth positive functions, and the former is subject to the following conditions

$$
\left.\vartheta\right|_{r<r_{h}}=10,\left.\quad \vartheta\right|_{r>r_{o}}=1, \quad \vartheta^{\prime} \leqslant 0
$$

For later use note a few simple properties of the functions $m(r), \epsilon(r)$ :

$$
\left.m(r)\right|_{r<r_{h}}=m_{0} r_{h}^{-10 / 3} r^{3},\left.\quad m(r)\right|_{r>r_{o}}=\text { const }
$$

and

$$
m^{\prime}(r)=\frac{(\vartheta-1) m(r)}{3 r} \geqslant 0, \quad\left[m^{\prime}(r) / r^{2}\right]^{\prime}=\frac{m(r)}{9 r^{4}}\left[3 r \vartheta^{\prime}+(\vartheta-1)(\vartheta-10)\right] \leqslant 0
$$

whence, in particular,

$$
\left.\epsilon\right|_{r<r_{h}}=\text { const, }\left.\quad \epsilon\right|_{r>r_{o}}=0 .
$$

We choose $m_{0}$ to be so small (by (A.3) it is always possible) that at $r \neq 0$

$$
r>2 m(r) \text { and hence } \epsilon^{\prime} \geqslant 0
$$

(to obtain the latter inequality, differentiate (A.2), use (A.4), and note that in the region where $\epsilon \neq 0$ (i. e. inside $B_{r_{o}}$ ) the upper limit of the integral (A.2) is less than the lower). 
Consider a (smooth by (A.3) and (A.6)) metric

$$
\mathrm{d} s^{2}=-e^{2 \epsilon}(1-2 m / r) \mathrm{d} t^{2}+(1-2 m / r)^{-1} \mathrm{~d} r^{2}+r^{2}\left(\mathrm{~d} \theta^{2}+\sin ^{2} \theta \mathrm{d} \phi^{2}\right) .
$$

Beyond $B_{r_{o}}$ ('outside the cluster') $\epsilon=0$ and $m=$ const, i. e. (A.7) becomes just the Schwarzschild metric. Let us check that for an appropriate choice of the free function $\varphi$ the metric (A.7) satisfies the weak energy condition. Using again equations (14.52) of [22] we find

$$
G^{\hat{0} \hat{0}}=2 r^{-2} m^{\prime} \geqslant 0
$$

Also

$$
G^{\hat{0} \hat{0}}+G^{\hat{1} \hat{1}}=2 \frac{r-2 m}{r^{2}} \epsilon^{\prime} \geqslant 0
$$

Finally, for $i=2,3$

$$
G^{\hat{0} \hat{0}}+G^{\hat{\imath} \hat{\imath}}=(1-2 m / r)\left[\epsilon^{\prime 2}+\frac{\epsilon^{\prime}}{r}\left(1-3 \frac{m^{\prime} r-m}{r-2 m}\right)+\epsilon^{\prime \prime}-\frac{r^{2}}{r-2 m}\left(m^{\prime} / r^{2}\right)^{\prime}\right] .
$$

The first term in the square brackets is nonnegative and so is the second term when $m_{0}$ is small enough. Hence (after twice differentiating (A.2)) we obtain

$$
G^{\hat{0} \hat{0}}+G^{\hat{\imath} \hat{\imath}} \geqslant(\varphi-1) \frac{r^{2}}{r-2 m}\left(m^{\prime} / r^{2}\right)^{\prime}
$$

The right hand side is nonnegative [see (A.4)] when $\varphi<1$.

Summing up, when $m_{0}$ is sufficiently small the metric (A.7) satisfies WEC for any $\varphi<$ 1. Moreover, it is easy to show that for some interval $\sigma \subset\left(r_{h}, r_{i}\right)$ the inequalities (A.8A.10) are strict. Thus WEC holds also for a metric (A.7) with $\varphi(r)$ replaced by a function $\varphi(r)-\kappa(t) \varphi_{1}(r)$, where $\kappa$ and $\varphi_{1}$ are non-negative, $\operatorname{supp} \varphi_{1} \subset \sigma$, and $\kappa, \dot{\kappa}, \ddot{\kappa}$ are sufficiently small. Consider such a metric in the case when $\kappa(t)$ grows (and, correspondingly, $\epsilon$, which by (A.5,A.6) is non-positive, grows too). In this metric, if a curve $\gamma \subset B$ is null, then any $\tilde{\gamma}$ obtained from $\gamma$ by a translation to the future in the $t$-direction will be timelike. In other words, the later one starts, the less time it will take to reach the destination, even though the metric outside $B_{r_{o}}$ remains Schwarzschild.

[1] M. Alcubierre, Class. Quantum Grav. 11, L73 (1994).

[2] S. Krasnikov, Phys. Rev. D 57, 4760 (1998). 
[3] A. E. Everett and T. A. Roman, Phys. Rev. D 56, 2100 (1997).

[4] D. H. Coule, Class. Quantum Grav. 15, 2523 (1998).

[5] M. J. Pfenning and L. H. Ford, Class. Quantum Grav. 14, 1743 (1997).

[6] M. S. Morris and K. S. Thorne, Am. J. Phys. 56, 395 (1988).

[7] H.-J. Schmidt, in: New frontiers in gravitation, Ed. G. Sardanashvily (Hadronic Press, 1996).

[8] K. Olum, Phys. Rev. Lett. 81, 3567 (1998).

[9] S. Gao and R. M. Wald, Class. Quantum Grav. 17, 4999 (2000).

[10] R. J. Low, Class. Quantum Grav. 16, 543 (1999).

[11] N. D. Birrel and P. C. V. Davies, Quantum Fields in Curved Space (Cambridge: Cambridge University Press, 1982).

[12] M. J. Pfenning, Quantum inequality restrictions on negative energy densities in curved spacetimes, gr-qc/9805037.

[13] L. H. Ford and T. A. Roman, Phys. Rev. D 55, 2082 (1997).

[14] L. H. Ford and T. A. Roman, Phys. Rev. D 53, 5496 (1996).

[15] D. N. Vollick, Phys. Rev. D 61, 084022 (2000); É. É. Flanagan, ibid. 66, 104007 (2002).

[16] C. J. Fewster and S. P. Eveson, Phys. Rev. D 58, 084010 (1998).

[17] K. D. Olum and N. Graham, Phys. Lett. B 554, 175 (2003).

[18] M. S. Morris, K. S. Thorne, and U. Yurtsever, Phys. Rev. Letters 61, 1446 (1988).

[19] S. Krasnikov, Phys. Rev. D 65, 064013 (2002).

[20] S. Krasnikov, Phys. Rev. D 62, 084028 (2000).

[21] M. Visser, Lorentzian wormholes — from Einstein to Hawking (New York, AIP Press, 1995).

[22] C. W. Misner, K. S. Thorne, and J. A. Wheeler, Gravitation (San Francisco, Freeman, 1973).

[23] C. Van Den Broeck, Class. Quantum Grav. 16, 3973 (1999). 